\title{
Mind the Mother When Considering Breastfeeding
}

\author{
Veronica Rivi ${ }^{1}$, Greta Petrilli ${ }^{2,3}$ and Johanna M. C. Blom ${ }^{1,4 *}$ \\ ${ }^{1}$ Department of Biomedical, Metabolic and Neural Sciences, University of Modena and Reggio Emilia, Modena, Italy, ${ }^{2}$ Unità \\ di Psichiatria-Ambulatorio Varenna per la Salute Mentale Perinatale, ASST Papa Giovanni XXIII, Bergamo, Italy, ${ }^{3}$ Area \\ Perinatale SIPRe-Milano, Società Italiana di Psicoanalisi della Relazione, Milan, Italy, ${ }^{4}$ Centre of Neuroscience and \\ Neurotechnology, University of Modena and Reggio Emilia, Modena, Italy
}

Keywords: maternal mental health, breastfeeding, perinatal period, screening, risk factors

\section{INTRODUCTION}

Depression and anxiety disorders represent the most common obstetric complications during pregnancy and the first-year post-partum, reducing the mother's ability to effectively perceive, decipher, and respond to their infant needs (1). An important question faced by mothers is whether to breastfeed their newborn child. Maternal mental health and breastfeeding have a tight but mixed relationship. Contrasting results have been reported in the literature (2): for each study demonstrating that breastfeeding is protective for the onset of anxiety and/or depression (3), there is a study showing that the difficulties related to breastfeeding can exacerbate the incidence and/or severity of maternal psychiatric illness $(4,5)$. Similarly, while several studies demonstrate a negative impact of depression on the duration of breastfeeding (6-8), others show that postpartum mental

OPEN ACCESS

Edited by: Adebola Emmanuel Orimadegun, University of Ibadan, Nigeria

Reviewed by:

Zahra Hoodbhoy, Aga Khan University, Pakistan Kwame Adu-Bonsaffoh University of Ghana, Ghana

*Correspondence: Johanna M. C. Blom joan.blom@unimore.it

Specialty section: This article was submitted to Maternal Health

a section of the journal Frontiers in Global Women's Health

Received: 17 June 2020

Accepted: 23 July 2020 Published: 15 September 2020

Citation:

Rivi V, Petrilli G and Blom JMC (2020) Mind the Mother When Considering Breastfeeding.

Front. Glob. Womens Health 1:3. doi: 10.3389/fgwh.2020.00003 illness could emerge as consequence of the early interruption of breastfeeding (9). Moreover, the all or none view that "breast is best" established by our society, makes some women feel inadequate if they choose not to breastfeed (10). Nevertheless, feeling inadequate or ashamed is not equivalent with suffering from anxiety or postpartum depression (PPD), and some women do not feel any negative pressure if they decline to breastfeed. There is no explicit reason, if a woman does not want to breastfeed, that her mental health and well-being depends on this decision or that she will experience any negative outcome as a result of her decision $(9,11)$. Given this and considering that breastfeeding recommendations are a critical and complex topic in public health discussions, our aim is to highlight various aspects surrounding breastfeeding with the purpose to propose to stably include screening for mental health in programs tailored to the mother.

\section{BREASTFEEDING AS MODERATOR}

Considering the numerous and complex biological, physiological, psychological, environmental, and sociocultural factors involved, breastfeeding can be defined at best as a moderator, decreasing or increasing anxiety, and depressive symptoms depending on the breastfeeding intentions of the mother $(9,11,12)$, her personal history, and any pre-existing or current maternal mental health and treatment related issues $(2,13)$. Each (breast)feeding experience, in fact, should be considered "with the mother in the mind," assuming that there is not a one-size-fits-all relationship between breastfeeding and maternal mental health. Successful breastfeeding starting immediately after birth and its continuation for the recommended or desired period is not the exclusive responsibility of the mother. It is a shared collective responsibility, with many stakeholders and moderating factors, among which the sociocultural environment, the context (family, workplace, healthcare system), and maternal physical and mental health (13-15).

Research routinely suggests that antenatal depression, considered in isolation, negatively influences breastfeeding. However, the effect of antenatal depression on breastfeeding should not 
be considered as a separate entity but is the result of many factors, such as the psychosocial and socio-economic context of the pregnant woman (11). Therefore, if we want to truly understand the relationship between antenatal anxiety and depression and breastfeeding, we should embrace a multidimensional approach to better understand the effects of the factors driving the intention to breastfeed which is set off by the mothers' state of mind. The main challenge is not to understand the role of each factor and context but the relationships and connectivity among them. New conceptual thinking will result in increasingly explanatory and predictive models which may offer a more realistic image of the strengths and vulnerabilities of pregnant women who intend to breastfeed by stressing the dynamic nature of these relationships. This type of approach may have important implications for clinical practice as it not only indicates which domains mostly influence antenatal depression, but also which factors are most central to the development of antenatal depression, and, therefore, may direct its overall effect on breastfeeding. As a consequence, we will be able to better recognize interrelated behaviors allowing to isolate the domain(s) most central to overall psychosocial risk, thus, improving successful breastfeeding while developing programs fundamental for the screening, surveillance and monitoring of antenatal depression and mental health more generally.

\section{IMPACT OF GENERAL POLICY}

Furthermore, it is important to be mindful that the policy of public health organizations, like the WHO, issued important recommendations promoting the concept that "breast is best" is based mainly on the need of third world countries for nutrition, and not on emotional well-being (16). Consequently, this policy is currently questioned extensively and a more inclusive belief of "fed is best" is put forward, involving women who breast-feed, bottle-feed or both and which stresses the first and foremost importance of providing nutrition avoiding at all cost, infant undernutrition. While more inclusive this approach still lacks a focus on the emotional well-being of the mother.

\section{THE BENEFITS AND POTENTIAL HARMS OF BREASTFEEDING}

Breastfeeding is more than just the delivery of the optimal infant nutrition, and offers a wide range of psychological benefits for both the infant and the mother, providing one of the earliest opportunities to facilitate maternal awareness of her infant needs and vice versa (17). The act of breastfeeding, in fact, promotes hormonal processes that induce the release of oxytocin, an important hormone related to maternal bonding, and attenuates the cortisol response to stress which when consistently high, is one of the strongest risk factors for the development of psychiatric disorders (17). Moreover, breastfeeding supports the regulation of sleep and wake patterns for both mother and infant and sustains and improves maternal self-efficacy (1). Consequently, breastfeeding mothers are more likely to report positive mood, less anxiety, and increased calm compared to formula feeding mothers $(1,8)$. Beyond the psychological benefits, breastfeeding provides substantial nutritional, cognitive, emotional, and immunologic benefits for the infants and their mothers. Breast milk, in fact, not only gives infants all the nutrients required for healthy development, but it also contains antibodies that protect infants from common childhood pathologies. Moreover, breastmilk affects the buildup of viral populations in the gut of the neonate which confers protection against harmful viruses and is thus fundamental in the interaction between the neonate and the microbial environment (18). For mothers, breastfeeding has proven to confer lower risk of breast and ovarian cancer, greater postpartum weight loss and decreased blood pressure compared with no breastfeeding (19).

In contrast, breastfeeding, which requires sustained mother-infant contact could represent a serious challenge for those mothers who suffer from perinatal mental diseases. Consequently, depressed mothers often report lower breastfeeding self-efficacy and tend to breastfeed less or for a shorter period of time $(2,6,9)$.

Even though women suffering from depression and anxiety during the perinatal period tend to be less likely to initiate or to maintain breastfeeding $(3,7,9)$, this does not preclude the possibility that breastfeeding has a positive effect on depressive and/or anxiety related symptoms during this period. In fact, some women who had no intention to breastfeed during gestation but changed their minds and attempted to breastfeed after all, showed a significant decrease in depressive symptoms from childbirth to 3 months postpartum $(2,9)$. Interestingly, other studies reported that mothers who do not suffer from anxiety and/or depression during pregnancy and do not initiate or maintain breastfeeding, are more at-risk for depression and/or anxiety in the postpartum period (9).

Also, breastfeeding has severe psychological consequences in women who were not depressed before delivery and intended to breastfeed but were not able to do so (10). Together these results are an ulterior demonstration of the complex and heterogeneous effects of breastfeeding on maternal mental health and suggest important implications for the way in which these mothers should be supported.

Although maternal depression and anxiety have been identified as risk factors for early breastfeeding cessation (3, 7), some studies showed that instead depression and anxiety could result as consequence of breastfeeding interruption $(1,2)$. Women who experience depression/anxiety and then proceed to breastfeed, may or may not experience further anxiety or depression even if they had early negative breastfeeding experiences. Thus, it is possible that mothers may have had an early negative breastfeeding experience (e.g., difficulty with latch-on, perceived insufficient milk, etc.) $(4,10)$ but have overcome these difficulties, with adequate support, and have moved forward to continue to breastfeed and in the end had a positive breastfeeding experience (successful latch-on, adequate or increased milk supply, etc.) accompanied by positive mental health outcomes (pride, increased breastfeeding self-efficacy, improved mood) as a result $(4,9)$. 


\section{Determinants of breastfeeding and maternal mental health}
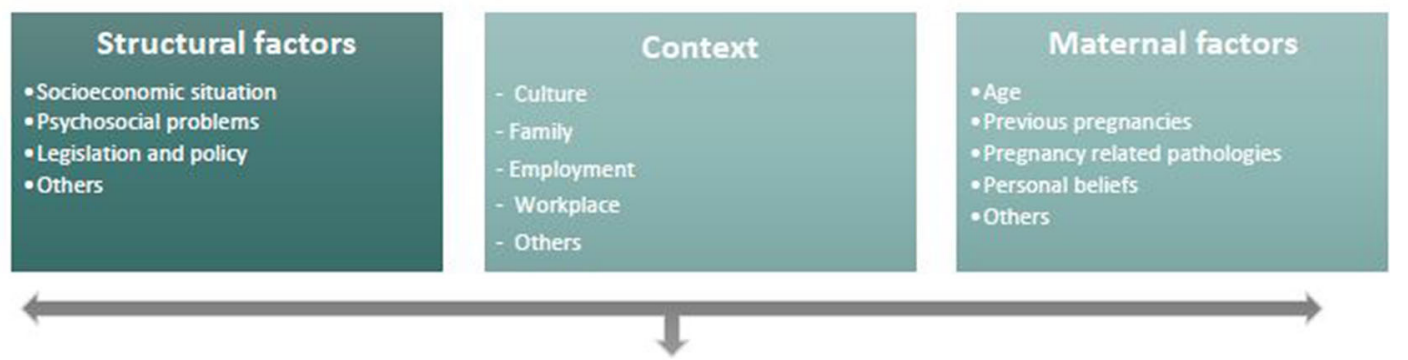

Intention to breastfeed

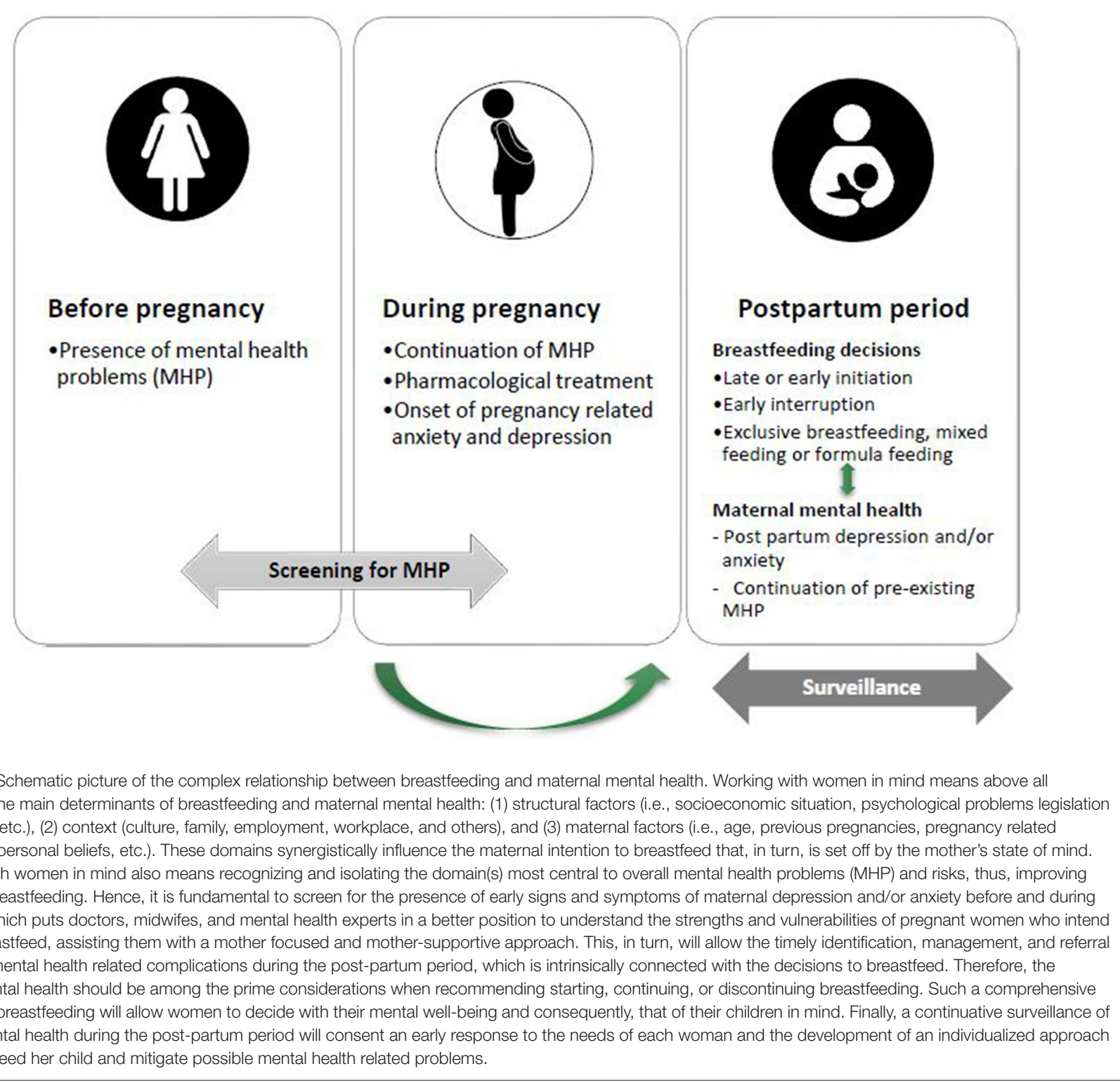




\section{OPERATING "WITH THE MOTHER IN THE MIND"}

Working "with the mother in the mind" also means to consider previous and current maternal life experiences (i.e., traumatic episodes, antidepressant treatments, age, parity, marital status, family income, social support, or maternal employment...) as significative factors influencing the perception of motherhood. In fact, when deciding whether to breastfeed, each new mother is influenced not only by her physical and psychological ability and personal beliefs, but also by social and cultural customs and norms. In those instances, in which breastfeeding improves maternal mental health this practice should be encouraged but when breastfeeding negatively affects maternal psychiatric symptoms, mothers should not be left to feel guilty if they choose to seek alternative forms of feeding. Given this, it is important that their partner and other family members participate in the decision related to the risks and benefits of breastfeeding and share the necessity to consider alternative options. Such considerations and inclusiveness could have a substantial positive impact on maternal mental health and may prevent further complications and negative outcomes for both the mothers and their children.

Maybe now is the moment we should look for a better more inclusive and multidisciplinary approach to the expectations, preferences, and decision-making of women regarding breastfeeding and revisit norms and recommendations that dominate conversations between the care team and women who are trying to decide how to feed their baby. First, and most importantly, the "breast is best" approach does not reflect the preference of all women and does not uniquely confer mental well-being. Also, many women resent that alternatives to breastfeeding are negatively framed which undermines confidence in them, to make the best choice for herself as well as for her baby. Instead, mothers prefer possibilistic, optimistic, or encouraging messages, ones that acknowledge and respect their choice and even anticipate the possibility of good outcomes.

A second important factor surrounding the choice to breastfeed is that it is often made in contexts in which the mother does not truly has a choice, such as when medical conditions of mother and neonate do not allow it. Perhaps in these situations

\section{REFERENCES}

1. Figueiredo B, Dias CC, Brandão S, Canário C, Nunes-Costa R. Breastfeeding and postpartum depression: state of the art review. J Pediatr. (2013) 89:332-8. doi: 10.1016/j.jped.2012.12.002

2. Pope CJ, Mazmanian D. Breastfeeding and postpartum depression: an overview and methodological recommendations for future research. Depress Res Treat. (2016) 2016:4765310. doi: 10.1155/2016/4765310

3. Figueiredo B, Canário C, Field T. Breastfeeding is negatively affected by prenatal depression and reduces postpartum depression. Psychol Med. (2014) 44:927-36. doi: 10.1017/S0033291713001530

4. Chaput KH, Nettel-Aguirre A, Musto R, Adair CE, Tough SC. Breastfeeding difficulties and supports and risk of postpartum depression in a cohort of the better approach would be to counsel and assist the mother, take away her uncertainties, anxieties, and doubts where possible and prepare her for alternatives conveying the idea that "fed is best" instead of "breast is best" (Figure 1).

\section{RECOMMENDATIONS}

\section{Screen and Surveil}

Routine pregnancy check-ups and postpartum visits (20) represent an ideal time for screening of the presence of early signs and symptoms of maternal depression and/or anxiety and screening tools that include simple questions regarding the intention to breastfeed are most informative and predictive of later problems.

\section{Identify}

Timely identification, management, and referral of mental health related complications in women during the perinatal period is fundamental and by implementing a multidisciplinary approach, which includes perinatal mental health experts, we will be better equipped to understand what mothers want by respecting her and demonstrating a non-judgmental mindset. To do that, we would need to listen more and better.

\section{Intervene and Prevent}

With this information, doctors, midwifes, and mental health experts, will be in a better position to understand the strengths and vulnerabilities of pregnant women who intend or not to breastfeed and intervene accordingly as maternal prenatal anxiety and depression are more readily affected by efficient treatment. Early response to the needs of each woman and the development of an individualized approach to how best feed her child will mitigate possible mental health related problems. In our opinion this is what most women want: a truly comprehensive approach to breastfeeding.

\section{AUTHOR CONTRIBUTIONS}

VR wrote the first draft. VR and GP performed the literature search. JB was responsible for the concept and the final draft of the paper. All authors contributed important scientific information to the paper, read, and approved the final paper for submission. Open. (2016) 4:E103-9. doi: 10.9778/cmajo.20150009

5. Dias CC, Figueiredo B. Breastfeeding and depression: a systematic review of the literature. J Affect Disord. (2015) 171:142-54. doi: 10.1016/j.jad.2014.09.022

6. Henderson JJ, Evans SF, Straton JAY, Priest SR, Hagan R. Impact of postnatal depression on breastfeeding duration. Birth Berkeley Calif. (2003) 30:175-80. doi: 10.1046/j.1523-536X.2003.00242.x

7. Hoff CE, Movva N, Rosen Vollmar AK, Pérez-Escamilla R. Impact of maternal anxiety on breastfeeding outcomes: a systematic review. Adv Nutr Bethesda Md. (2019) 10:816-26. doi: 10.1093/advances/nmy132

8. Hahn-Holbrook J, Haselton MG, Dunkel Schetter C, Glynn LM. Does breastfeeding offer protection against maternal depressive 
symptomatology? Arch Womens Ment Health. (2013) 16:411-22. doi: 10.1007/s00737-013-0348-9

9. Borra C, Iacovou M, Sevilla A. New evidence on breastfeeding and postpartum depression: the importance of understanding women's intentions. Matern Child Health J. (2015) 19:897-907. doi: 10.1007/s10995-014-1591-z

10. McIntyre LM, Griffen AM, BrintzenhofeSzoc K.. Breast is best... except when it's not. J Hum Lact. (2018) 34:575-80. doi: 10.1177/0890334418774011

11. Amiel Castro RT, Glover V, Ehlert U, O'Connor TG. Antenatal psychological and socioeconomic predictors of breastfeeding in a large community sample. Early Hum Dev. (2017) 110:50-6. doi: 10.1016/j.earlhumdev.2017.04.010

12. Jones CL, Culpin I, Evans J, Pearson RM. Relative effects of breastfeeding intention and practice on maternal responsiveness. Infant Ment Health J. (2020) 41:82-93. doi: 10.1002/imhj.21832

13. Galbally M, Watson S, Boyce P, Nguyen T, Lewis A. The mother, the infant and the mother-infant relationship: what is the impact of antidepressant medication in pregnancy. J Affect Disord. (2020) 272:363-70. doi: 10.1016/j.jad.2020.03.116

14. Victora CG, Bahl R, Barros AJ, França GV, Horton S, Krasevec J, et al. Breastfeeding in the 21st century: epidemiology, mechanisms, and lifelong effect. Lancet. (2016) 387:475-90. doi: 10.1016/S0140-6736(15)01024-7

15. Rollins NC, Bhandari N, Hajeebhoy N, Horton S, Lutter CK, Martines JC, et al. Why invest, and what it will take to improve breastfeeding practices? Lancet. (2016) 387:491-504. doi: 10.1016/S0140-6736(15)01044-2

16. WHO. 3 in 5 Babies Not Breastfed in the First Hour of Life. Available online at: https://www.who.int/news-room/detail/31-07-2018-3-in-5-babies-notbreastfed-in-the-first-hour-of-life
17. Mezzacappa ES, Katkin ES. Breast-feeding is associated with reduced perceived stress and negative mood in mothers. Health Psychol. (2002) 21:18793. doi: $10.1037 / 0278-6133.21 .2 .187$

18. Liang G, Zhao C, Zhang H, Mattei L, Sherrill-Mix S, Bittinger K, et al. The stepwise assembly of the neonatal virome is modulated by breastfeeding. Nature. (2020) 581:470-4. doi: 10.1038/s41586-020-2192-1

19. Chowdhury R, Sinha B, Sankar MJ, Taneja S, Bhandari N, Rollins N, et al. Breastfeeding and maternal health outcomes: a systematic review and meta-analysis. Acta Paediatr. (2015) 104:96-113. doi: 10.1111/apa. 13102

20. Wouk K, Stuebe AM, Meltzer-Brody S. Postpartum mental health and breastfeeding practices: an analysis using the 20102011 pregnancy risk assessment monitoring system. Matern Child Health J. (2017) 21:636-47. doi: 10.1007/s10995-016-2 $150-6$

Conflict of Interest: The authors declare that the research was conducted in the absence of any commercial or financial relationships that could be construed as a potential conflict of interest.

Copyright (c) 2020 Rivi, Petrilli and Blom. This is an open-access article distributed under the terms of the Creative Commons Attribution License (CC BY). The use, distribution or reproduction in other forums is permitted, provided the original author(s) and the copyright owner(s) are credited and that the original publication in this journal is cited, in accordance with accepted academic practice. No use, distribution or reproduction is permitted which does not comply with these terms. 